\title{
Complicaciones quirúrgicas del linfoma con compromiso gastrointestinal
}

\author{
Surgical complications of lymphoma with gastrointestinal involvement \\ Juliana Lucia Molina-Valencia' $\mathbb{D}$, Carlos Andrés Delgado-López² $\mathbb{D}$ \\ 1 Médica, residente de Cirugía General, departamento de Cirugía General, Universidad de Antioquia, Medellín, Colombia. \\ 2 Médico, especialista en Cirugía General, Hospital Pablo Tobón Uribe, Medellín, Colombia.
}

\section{Resumen}

Introducción. El linfoma puede afectar el tracto gastrointestinal de manera primaria o secundaria, y representa hasta el $2 \%$ de todas las neoplasias malignas del intestino delgado y colon. El tracto gastrointestinal es la ubicación extraganglionar primaria más común en el linfoma no Hodgkin.

Métodos. Se realizó una búsqueda de la literatura en las principales bases de datos académicas, con revisión de textos publicados sobre el tema en los últimos 5 años.

Discusión. La presentación clínica del linfoma con compromiso gastrointestinal es inespecífica y, hasta en la mitad de los pacientes, se puede presentar de manera inicial con complicaciones que requieran manejo quirúrgico. Entre las principales se encuentran la perforación intestinal, el sangrado digestivo y la obstrucción intestinal.

Palabras clave: linfoma; tracto gastrointestinal; neoplasias gastrointestinales; obstrucción intestinal; perforación intestinal; intususcepción.

\begin{abstract}
Introduction. Lymphoma can affect the gastrointestinal tract, primarily or secondarily, and accounts for up to $2 \%$ of all malignant neoplasms of the small intestine and colon. The gastrointestinal tract is the most common primary extranodal location in non-Hodgkin lymphoma.

Methods. A literature search performed in the main academic databases, with a review of texts published on the subject in the last 5 years.

Discussion. The clinical presentation of lymphoma with gastrointestinal involvement is nonspecific and, in up to half of the patients, it can present initially with complications that require surgical management. Among the main ones are intestinal perforation, digestive bleeding and intestinal obstruction.
\end{abstract}

Keywords: lymphoma; gastrointestinal tract; gastrointestinal neoplasms; intestinal obstruction; intestinal perforation; intussusception.

Fecha de recibido: 08/09/2020 - Fecha de aceptación: 30/01/2021 - Fecha de publicación en línea: 05/04/2021

Correspondencia: Juliana Lucia Molina-Valencia, Calle 70 No. 52-21, Medellín, Colombia. Tel: (+57) 3013163505

Correo electrónico: juliana.molina@udea.edu.co

Citar como: Molina-Valencia JL, Delgado-López CA. Complicaciones quirúrgicas del linfoma con compromiso gastrointestinal. Rev Colomb Cir. 2021;36:514-9. https://doi.org/10.30944/20117582.764

Este es un artículo de acceso abierto bajo una Licencia Creative Commons - BY-NC-ND https://creativecommons.org/licenses/by-ncnd/4.0/deed.es 


\section{Introducción}

El epitelio intestinal y la secreción mucosa son la primera línea de defensa contra patógenos en el tracto gastrointestinal, pero una respuesta inmunológica más específica se encuentra a cargo del tejido linfoide asociado a la mucosa (MALT; por sus siglas en inglés) ${ }^{1}$. La respuesta inflamatoria y la estimulación antigénica repetitiva pueden llevar a una proliferación monoclonal y al desarrollo de un linfoma ${ }^{2,3}$.

El compromiso gastrointestinal por linfoma representa un grupo de diferentes entidades clínico-patológicas de células B (90\%) o T (10\%). La afectación gastrointestinal primaria del linfoma no Hodgkin es la ubicación extraganglionar más frecuente, causando un $30 \%$ a $40 \%$, mientras el compromiso secundario se reporta entre el $10 \%$ a $15 \%{ }^{4}$. El linfoma Hodgkin con afectación gastrointestinal es extremadamente raro ${ }^{5}$.

El linfoma gastrointestinal primario afecta principalmente el estómago, seguido de intestino delgado, colon, recto, afectación múltiple, y raramente, esófago. El linfoma representa el $0,2 \%$ al $2 \%$ de todas las neoplasias malignas del intestino delgado y colon, típicamente se presenta en la sexta década de la vida y es un poco más frecuente en hombres ${ }^{6,7}$.

El papel de las intervenciones quirúrgicas ha cambiado con los años. La cirugía es fundamental cuando se presenta alguna complicación como perforación intestinal, obstrucción intestinal o sangrado digestivo, documentadas hasta en el $20 \%$ de los pacientes con linfoma con compromiso gastrointestinal ${ }^{4,5}$. La literatura disponible desde el enfoque quirúrgico no es extensa, por lo cual, el objetivo de esta revisión fue describir las principales complicaciones de interés para el cirujano.

\section{Métodos}

Se realizó una búsqueda de la literatura sobre artículos publicados desde enero de 2015 hasta enero de 2020 en bases de datos como PudMed, LILACS y Google académico, con las palabras clave: linfoma, dolor abdominal, perforación intestinal, obstrucción intestinal, intususcepción. Se revi- saron artículos en español, inglés y francés. Se incluyeron todo tipo de publicaciones.

\section{Discusión}

Los linfomas se deben distinguir en su origen celular y presentación clínica para determinar su evolución y tratamiento. Los signos y síntomas pueden superponerse en la población, con y sin complicaciones. Entre $45 \%$ y $65 \%$ se presentan con dolor abdominal crónico, intermitente, asociado a náuseas y emesis, distensión abdominal, constipación o diarrea. Los síntomas constitucionales, como fiebre y pérdida de peso, son menos comunes y, en la mayoría de los casos, no existe el antecedente de enfermedad linfoproliferativa.

Entre los factores de riesgo para su desarrollo se encuentran infección por Helicobacter pylori, enfermedad celiaca, enfermedad inflamatoria intestinal, inmunosupresión, infección por el virus de la inmunodeficiencia humana (VIH) o trasplante de órgano sólido ${ }^{8,9}$.

Con frecuencia, el diagnóstico se hace después de una intervención quirúrgica, cuando se presenta una complicación, siendo las más frecuentes perforación intestinal, obstrucción y sangrado ${ }^{1,10-12}$.

Los diagnósticos diferenciales por imagen son principalmente el adenocarcinoma y los tumores estromales. Un compromiso generalizado o multifocal intestinal, la ausencia de obstrucción a pesar de gran volumen tumoral y la ausencia de hipervascularización, son señales que apuntan hacia un linfoma. En el colon, particularmente en el ciego, y el recto, las lesiones pueden ser masa polipoide, infiltración circunferencial, masa ulcerada, haustras engrosadas o nódulos mucosos ${ }^{13,14}$.

Hasta el momento no existe una escala validada para predecir el riesgo de complicaciones en los pacientes con linfoma gastrointestinal. En el estudio de Aoki et al. ${ }^{5}$, describieron que en el $48 \%$ de los pacientes la presentación inicial fue por una de las complicaciones y en $52 \%$ las complicaciones se desarrollaron posterior al inicio de la quimioterapia. Este mismo grupo evaluó los factores de riesgo de requerir cirugía por complicaciones gastrointestinales del linfoma 
y propusieron un puntaje de riesgo, que por ahora, solo cuenta con validación interna, denominado el "FLASH score" que incluye: A: Apariencia focal y tamaño mayor o igual de $40 \mathrm{~mm}$ (1 punto); B: Linfoma del intestino delgado con histología agresiva (2 puntos); C: Alta captación de las lesiones en la Tomografía de positrones con 18F-fluorodesoxiglucosa (1 punto). Los pacientes con 4 puntos tenían un riesgo de $69 \%$ de eventos gastrointestinales, con un área bajo la curva de 0,91 $\left(\mathrm{IC}_{95 \%} 0,86-0,97\right)$.

\section{Perforación intestinal}

La perforación intestinal es una de las complicaciones más conocidas del linfoma no Hodgkin, presente del 1 al $25 \%$ de los linfomas con compromiso intestinal, relacionada o no a la quimioterapia ${ }^{15}$. En una serie reportada de 1062 pacientes, $9 \%$ presentaron perforación intestinal luego del inicio de la quimioterapia, la media fue de 46 días, más común en intestino delgado, subtipo linfoma difuso de células B grandes (DLBCL, por sus siglas en inglés) ${ }^{16,17}$. Las razones para la perforación en pacientes que reciben quimioterapia son la rápida necrosis del tumor, lisis tumoral y alteraciones del tejido por la rápida granulación. La tasa de perforación en linfomas de células T es mayor (41 - 50 \%) que en células B (30\%), por su afectación de la mucosa con invasión de paredes vasculares llevando a isquemia y necrosis ${ }^{18,19}$.

El manejo quirúrgico electivo con resección del segmento intestinal afectado por linfoma se ha propuesto como una estrategia para evitar las complicaciones posteriores al inicio de la quimioterapia, ya que una intervención urgente por perforación se asocia a un riesgo aumentado de morbimortalidad. Sin embargo, esta práctica se ha abandonado en los últimos años pues no ha demostrado mejorar tasas de supervivencia a tres y cinco años ${ }^{17,20 .}$

Otra estrategia que se ha usado es hospitalizar a los pacientes después del primer ciclo de quimioterapia para implementar reposo intestinal con nutrición parenteral total. Esta práctica fue evaluada por Chin et al. ${ }^{16}$, de manera retrospectiva en 414 pacientes, sin encontrar diferencias en las tasas de perforación, peritonitis, cirugía o supervivencia global, pero si hubo un aumento importante en los costos. En este estudio los factores de riesgo relacionados con perforación fueron una pobre clase funcional, compromiso del intestino delgado o colon y enteropatía asociada a linfoma de células $\mathrm{T}$.

Los signos clínicos, como la irritación peritoneal, pueden estar enmascarados de manera inicial en los pacientes con quimioterapia, principalmente por las dosis altas de prednisolona ${ }^{21}$. La tomografía computarizada de abdomen con contraste es la ayuda más usada para determinar el diagnóstico y manejo. Una vez ha ocurrido esta complicación, durante la cirugía se practica resección del segmento perforado y anastomosis intestinal, siempre que las condiciones del paciente lo permitan, y posteriormente debe continuar con seguimiento y manejo por hemato-oncología ${ }^{22,23}$.

\section{Obstrucción intestinal e intususcepción}

La ausencia de reacción desmoplásica hace que la presentación del linfoma con obstrucción intestinal sea rara, a diferencia de lo que ocurre en caso de adenocarcinoma. Usualmente tiene un curso clínico intermitente de dolor abdominal tipo cólico, distensión abdominal y emesis, posiblemente relacionado con episodios de obstrucción intestinal subaguda con resolución espontánea ${ }^{24,25}$.

Para el estudio de la obstrucción intestinal, la tomografía computarizada de abdomen con contraste intravenoso tiene una sensibilidad de 94 a $100 \%$ y una especificidad de 90 a $95 \%{ }^{18,26}$. Es posible encontrar engrosamiento de la pared, lesiones infiltrativas o polipoides, dilatación aneurismática y conglomerados de ganglios linfáticos que disminuyen la luz intestinal ${ }^{27}$.

La obstrucción se puede presentar secundaria a una estenosis intra o extraluminal. Hasta el $54 \%$ de los linfomas de ubicación ileocecal requieren intervención quirúrgica, comúnmente por intususcepción ${ }^{24,28}$. En adultos la obstrucción intestinal por intususcepción representa del 1 al $5 \%$ de todas las causas mecánicas de obstrucción. En niños es una causa más frecuente de obstrucción intestinal, ya que el $90 \%$ de los casos son idiopáticos, 
a diferencia de la población adulta, donde la mayoría tienen una patología que predispone a la intususcepción al generar un punto de fijación en un segmento intestinal ${ }^{7,29}$. En el colon la intususcepción es secundaria a un tumor maligno en el 65 al $70 \%$ de los casos, mientras que en el intestino delgado del 30 al $35 \%$ se relacionan con malignidad $^{30,31}$.

En la tomografía computarizada, en los cortes axiales se observa una lesión con densidad de partes blandas intraluminal y densidad grasa periférica, debida al mesenterio invaginado (imagen en diana) y, en los cortes coronales, se aprecia una imagen de "pseudoriñon" ${ }^{32}$. En el estudio de Al-Radaideh et al. se encontró, en pacientes con historia conocida de linfoma, que la intususcepción también puede ser un hallazgo incidental o transitorio, con imágenes características de una localización proximal y longitud del segmento menor de $4 \mathrm{~cm}$, con resolución en estudios imagenológicos posteriores ${ }^{30,33}$.

El tratamiento óptimo de la intususcepción intestinal no se ha acordado de manera universal. Se recomienda la resección quirúrgica, y no únicamente la reducción, por la dificultad para diferenciar previo a la cirugía lesiones benignas de malignas. Cuando el diagnóstico de linfoma es conocido y el compromiso es difuso, la controversia en el manejo es mayor, sin embargo, se favorece la resección del segmento invaginado por el riesgo de necrosis y perforación ${ }^{29}$. Adicionalmente, la reducción previa a la resección no se recomienda, por aumentar el riesgo de perforación intestinal, implantación intraluminal y embolización de células malignas durante la manipulación. Solo se recomendaría la reducción sin resección en pacientes con riesgo de intestino corto ${ }^{34}$.

\section{Sangrado gastrointestinal}

El sangrado gastrointestinal por linfoma es raro y el sitio más común de sangrado es el estómago. Clínicamente, la mayoría de los pacientes presentan epigastralgia, dispepsia, hematemesis y melenas. Aproximadamente, el 20 a $30 \%$ de los DLBCL gástricos se presentan con sangrado ${ }^{35,36}$.

En la endoscopia se puede encontrar ulceración, pliegues gástricos engrosados, infiltración difusa, masa polipoide y cambios hiperémicos de la mucosa, que no son hallazgos específicos. Adicional a la biopsia de la lesión, se debe tomar biopsias del antro, cuerpo, duodeno y unión esófago-gástrica, y siempre buscar $H$. pylori, ya que el linfoma MALT se relaciona en un $75 \%$ con esa infección, al activar linfocitos T CD4 y estimular linfocitos $\mathrm{B}^{37}$. Más del $70 \%$ de los pacientes con estadio tempranos de linfoma MALT (Ann Arbor I - II) asociado a infección por $H$. pylori pueden lograr remisión con terapia de erradicación.

Según los hallazgos en la endoscopia, se pueden usar varias herramientas para control del sangrado, que no difieren a las utilizadas en el manejo de otras causas de sangrado, como fulguración con argón plasma, clips y escleroterapia ${ }^{35}$. El manejo definitivo del linfoma dependerá de la histología y el estadio.

Kadota et al. reportaron en pacientes con lesiones gástricas por DLBCL, que la progresión y no respuesta de la enfermedad a la quimioterapia se relacionó con complicaciones como el sangrado, perforación y estenosis. Los niveles de albumina menores de 3,4 g/dl se asociaron de manera independiente con estas complicaciones. Hasta el $71 \%$ de los sangrados gástricos se presentan durante el primer ciclo de quimioterapia y usualmente se resuelve con manejo endoscópico. La gastrectomía en estos pacientes no mejora la supervivencia, aunque en ocasiones es necesaria para el control del sangrado ${ }^{36}$.

\section{Conclusiones}

El linfoma representa hasta el $2 \%$ de todas las neoplasias malignas de intestino delgado y colon. Puede comprometer el tracto gastrointestinal de manera primaria o secundaria, siendo este último más frecuente. El linfoma gástrico se comporta de manera diferente a los linfomas del intestino delgado y colon, con una mayor supervivencia.

Se han reportado tasas de complicaciones que requieren cirugía urgente del 11 al $20 \%$ de los pacientes con linfoma, por obstrucción, perforación o sangrado, como causas más frecuentes. El tratamiento de elección para la mayoría de los linfomas con compromiso gastrointestinal es la quimioterapia. 
Actualmente la recomendación, sin una evidencia fuerte a favor (por la heterogeneidad de los resultados en diferentes estudios), es la indicación de manejo quirúrgico únicamente cuando se presente alguna complicación que lo amerite. Las resecciones quirúrgicas extensas comparadas con la resección segmentaria no dan beneficio en supervivencia.

\section{Cumplimiento de normas éticas}

Consentimiento informado: Este estudio es una revisión de la literatura, y como tal no hay necesidad de un consentimiento informado ni de aprobación del Comité de Ética Institucional.

Conflictos de interés: Los autores no reportaron ningún conflicto de intereses.

Fuentes de financiación: autofinanciado por los autores.

\section{Contribución de los autores:}

- Juliana Lucia Molina: concepción y diseño del artículo, obtención de los datos y su interpretación, redacción del trabajo, aportes intelectuales relevantes, revisión crítica y aprobación final.

- Carlos Andrés Delgado: concepción y diseño del artículo, obtención de los datos y su interpretación, redacción del trabajo, aportes intelectuales relevantes, revisión crítica y aprobación final.

\section{Referencias}

1. Shirwaikar TA, Schwartz M, Quigley E. Gastrointestinal lymphoma: the new mimic. BMJ Open Gastro. 2019;6:e000320.

https://doi.org/10.1136/bmjgast-2019-000320

2. Hustings N, Feyaerts F. Large B-cell Lymphoma of the Jejunum. J Belg Soc Radiol. 2019;103:41. https://doi.org/10.5334/jbsr.1826

3. Kohri M, Tsukasaki K, Akuzawa Y, Tanae K, Takahashi N, Saeki T, et al. Peripheral T-cell lymphoma with gastrointestinal involvement and indolent T-lymphoproliferative disorders of the gastrointestinal tract. Leukemia Research. 2020;91:106336. https://doi.org/10.1016/j.leukres.2020.106336

4. Matysiak-Budnik T, Fabiani B, Hennequin C, Thieblemont C, Malamut G, Cadiot G, et al. Gastrointestinal lymphomas: French Intergroup clinical practice recommendations for diagnosis, treatment and follow-up (SNFGE, FFCD, GERCOR, UNICANCER, SFCD, SFED, SFRO, SFH). Dig Liver Dis. 2018;50:124-131. https://doi.org/10.1016/j.dld.2017.12.006

5. Aoki T, Yamada A, Takahashi M, Niikura R, Toyama K, Ushiku T, et al. Development and internal validation of a risk scoring system for gastrointestinal events requiring surgery in gastrointestinal lymphoma patients. J Gastroenterol Hepatol. 2019;34:693-9. https://doi.org/10.1111/jgh.14452

6. Lightner AL, Shannon E, Gibbons MM, Russell MM. Primary gastrointestinal non-Hodgkin's lymphoma of the small and large intestines: A systematic review. J Gastrointest Surg. 2016;20:827-39. https://doi.org/10.1007/s11605-015-3052-4

7. Corti M, Boschi A, Portillo Á, Méndez N, Campitelli A, Narbaitz M. Invaginación intestinal como forma de presentación de linfoma no Hodgkin primario de colon en un paciente VIH positivo. Rev Esp Enferm Dig. 2016;108:750-3. https://doi.org/10.17235/reed.2016.3965/2015

8. Facundo-Navia H, Manrique-Acevedo ME. Linfoma primario del intestino delgado:reporte de un caso y revisión de la literatura. Rev Colomb Gastroenterol. 2017;32:6571. https://doi.org/10.22516/25007440.132

9. Wang Y, Li Y, Meng X, Duan X, Wang M, Chen W, et al. Epstein-Barr virus-associated T-cell lymphoproliferative disorder presenting as chronic diarrhea and intestinal bleeding: a case report. Front Immunol. 2018;9:2583. https://doi.org/10.3389/fimmu.2018.02583

10. Rehaman SA, Ramachandra CS, Jackaya RP. Primary gastro intestinal lymphoma presenting as perforation peritonitis. J Clin Diagn Res. 2016;10:PD22-4. https://doi.org/10.7860/JCDR/2016/15462.7483

11. Iida T, Nozawa H, Sonoda H, Toyama K, Kawai K, Hata K, et al. Upfront surgery for small intestinal non-Hodgkin's lymphoma. Anticancer Res. 2020;40:2373-7. https://doi.org/10.21873/anticanres.14206

12. Wang YG, Zhao LY, Liu CQ, Pan SC, Chen XL, Liu K, et al. Clinical characteristics and prognostic factors of primary gastric lymphoma: A retrospective study with 165 cases. Medicine. 2016;95:e4250. https://doi.org/10.1097/MD.0000000000004250

13. Sokhandon F, Al-Katib S, Bahoura L, Copelan A, George D, Scola D. Multidetector CT enterography of focal small bowel lesions: a radiological-pathological correlation. Abdom Radiol. 2017;42:1319-41. https://doi.org/10.1007/s00261-016-1015-1

14. Inada R, Fukata N, Ito T, Hamada M. A case of aneurysmal lymphoma of the small intestine. Jpn J Clin Oncol. 2016;46:288-9. https://doi.org/10.1093/jjco/hyw019

15. Isayama $C$, Takeuchi $N$, Takada $M$, Nishida $Y$, Nomura $Y$, Iida M, Otsuka A. Primary T-cell lymphoma with small intestinal perforation: A case report. J Med Cases. 2016;7:539-42. https://doi.org/10.14740/jmc2690w

16. Chin CK, Tsang E, Mediwake H, Khair W, Biccler J, Hapgood G, et al. Frequency of bowel perforation and impact of bowel rest in aggressive non-Hodgkin lymphoma with gastrointestinal involvement. Br J Haematol. 2019;184:826-8. https://doi.org/10.1111/bjh.15173 
17. Imataki O, Shiroshita K, Uchida S, Kida J, Akamoto S, Uemura M. Perforation in an intestinal malignant lymphoma case. BMC Res Notes. 2016;9:308. https://doi.org/10.1186/s13104-016-2111-6

18. Karadeli E, Erbay G, Parlakgumus A, Yabanoglu H. Abdominal CT findings in patients with primary lymphoma causing small bowel obstruction. J Coll Physicians Surg Pak. 2017;27:711-713. https://doi.org/10.1594/ecr2017/C-1229

19. Wong YJ, Lum HM, Fook-Chong S, Lim ST, Salazar E. Do total parenteral nutrition and bowel rest reduce the risk for perforation in patients with gastrointestinal tract lymphoma receiving chemotherapy? Nutrition. 2019;67-68:110515. https://doi.org/10.1016/j.nut.2019.03.023

20. Lu YH, Chang ST, Yang SF, Weng SF, Huang WT, Hsieh PP, et al. Primary intestinal diffuse large B-cell lymphoma in Taiwan showed a relative higher rate of perforation and EBV association. Appl Immunohistochem Mol Morphol 2015;24:1-9. https://doi.org/10.1097/PAI.0000000000000226

21. Tatar C, Yavas M, Akkus O, Tapkan B, Batikan OK, Bayrak $\mathrm{S}$, Arikan S. Intestinal perforation that developed after chemotherapy in a patient diagnosed with non-Hodgkin lymphoma: A case report and review of literature. Int J Surg Case Rep. 2017;39:321-3. https://doi.org/10.1016/j.ijscr.2017.08.058

22. Abbott $S$, Nikolousis E, Badger I. Intestinal lymphoma--a review of the management of emergency presentations to the general surgeon. Int J Colorectal Dis. 2015;30:151-7. https://doi.org/10.1007/s00384-014-2061-1

23. Singh A, Prabhakar G. Traumatic rupture of jejunal Burkitt's lymphoma with intestinal transection. J Indian Assoc Pediatr Surg. 2019;24:61-2. https://doi.org/10.4103/jiaps.JIAPS_67_18

24. Sudworth O, Hameed A. Delayed diagnosis of a high-grade B-cell non-Hodgkin's lymphoma presenting as an obstructing rectal mass. BMJ Case Rep. 2015;2015:bcr2015213146. https://doi.org/10.1136/bcr-2015-213146

25. Nehme F, Alderson J, Nassif I. Small bowel obstruction secondary to ileal mucosa-associated lymphoid tissue lymphoma. J Gastrointest Cancer. 2018;49:207-10. https://doi.org/10.1007/s12029-016-9882-9

26. Zaarour M, Busack C, Munker R. Obstructing duodenal diffuse large B-cell lymphoma with peritoneal lymphomatosis with exceptional response to R-CHOP. Cureus. 2019;11:e4621. https://doi.org/10.7759/cureus.4621

27. Leshchinskiy S, Ali N, D'Agostino R. The small bowel "aneurysm". Abdom Radiol. 2018;43:3503-4. https://doi.org/10.1007/s00261-018-1605-1
28. Waisberg J, Anderi ADV, Cardoso PAS, Borducchi JHM, Germini DE, Franco MIF, et al. Extensive colorectal lymphomatous polyposis complicated by acute intestinal obstruction: a case report. J Med Case Rep. 2017;11:190.

https://doi.org/10.1186/s13256-017-1340-1

29. Yang TW, Lin YY, Tsuei YW, Chen YL, Huang CY, Hsu SD. Successful management of adult lymphoma-associated intussusception by laparoscopic reduction and appendectomy. World J Gastroenterol. 2016;22:4781-5. https://doi.org/10.3748/wjg.v22.i19.4781

30. Al-Radaideh AM, Omari HZ, Bani-Hani KE. Adult intussusception: A 14-year retrospective study of clinical assessment and computed tomography diagnosis. Acta Gastroenterol Belg. 2018;81:367-72.

31. İflazoğlu N, Mengeloğlu A, Korkmaz NS, Karaalioğlu B, Yülüklü M. Primary small bowel lymphoma presenting as invagination. Turk J Surg. 2018;34:331-3. https://doi.org/10.5152/turkjsurg.2017.3115

32. Valentini V, Buquicchio GL, Galluzzo M, Ianniello $\mathrm{S}$, Di Grezia G, Ambrosio R, et al. Intussusception in adults: The role of MDCT in the identification of the site and cause of obstruction. Gastroenterol Res Pract. 2016;(11):5623718. https://doi.org/10.1155/2016/5623718

33. Negoi I, Paun S, Hostiuc S, Stoica B, Tanase I, Negoi RI, Beuran M. Most small bowel cancers are revealed by a complication. Einstein (Sao Paulo). 2015;13:500-5. https://doi.org/10.1590/S1679-45082015A03380

34. El-Sergany A, Darwish A, Mehta P, Mahmoud A. Community teaching hospital surgical experience with adult intussusception: Study of nine cases and literature review. Int J Surg Case Rep. 2015;12:26-30. https://doi.org/10.1016/j.ijscr.2015.03.032

35. Kim MS, Park JH, Kim JY, Lim SN, Lee J. Upper gastrointestinal bleeding caused by direct invasion of diffuse large B-cell lymphoma into the stomach in a patient with HIV infection: A case report. Medicine (Baltimore). 2019;98:e16363. https://doi.org/10.1097/MD.0000000000016363

36. Kadota T, Seo S, Fuse H, Ishii G, Itoh K, Yano T, et al. Complications and outcomes in diffuse large B-cell lymphoma with gastric lesions treated with R-CHOP. Cancer Med. 2019;8:982-9. https://doi.org/10.1002/cam4.1982

37. Juárez-Salcedo LM, Sokol L, Chavez JC, Dalia S. Primary gastric lymphoma, epidemiology, clinical diagnosis, and treatment. Cancer Control. 2018;25: 10732 74818778256. https://doi.org/10.1177/1073274818778256 